\title{
Assessing The Feasibility Of Pedometers For Quantifying The Volume Of Impacts During Varsity Athletic Practices
}

\author{
Timothy A. Burkhart (Corresponding author) \\ Department of Mechanical and Materials Engineering \\ 1151 Richmond Rd, London ON, N6A 5B9, Canada \\ Tel: 1-519-661-2111 X:81272 E-mail: tburkhar@uwo.ca \\ Robyn A. Bertram \\ bSchool of Rehabilitation Sciences, McMaster University \\ 1280 Main St. Hamilton, ON, L8S 4L8 \\ Alison Schinkel-Ivy \\ cSchool of Kinesiology and Health Sciences, York University, \\ 4700 Keele Street, Toronto ON, M3J 1P3 \\ David M. Andrews \\ Department of Kinesiology, University of Windsor \\ 401 Sunset Ave, Windsor ON, Canada N9B 3P4
}

Received: 19-11- 2013

doi:10.7575/aiac.ijkss.v.2n.1p.7
Accepted: 08-01- 2014

Published: 31-01- 2014

URL: http://dx.doi.org/10.7575/aiac.ijkss.v.2n.1p.7

The research is financed by the Natural Sciences and Research Council (NSERC) of Canada

\begin{abstract}
The purpose of the current investigation was to test the feasibility of utilizing pedometer data collected during gamelike practices to quantify the number of impacts experienced by varsity athletes. Forty-four varsity basketball and soccer athletes wore pedometers and the total number of steps and practice time were recorded during two different practices of similar intensity. The normalized step count, calculated as the total numbers of steps divided by the practice time, was obtained from the first practice and was used to estimate the step count for the second practice. The estimated step count was then compared to the actual step count, as determined from the pedometers. The mean percent difference between actual and estimated step counts was under $25 \%$ for approximately $75 \%$ of all athletes with no significant difference between the estimated and actual number of steps. The presented results suggest that the pedometer-based method presented here is a feasible method for estimating the number of steps experienced by university varsity athletes.
\end{abstract}

Keywords: Impact Injuries, Step Number, Exposure Quantification

\section{Introduction}

Repetitive impacts to the lower extremity during activities such as running and jumping can result in forces that exceed three times body weight (Nigg, Cole \& Bruggeman, 1995). Impacts such as these have been associated with overuse injuries including cartilage degeneration (Serink, Nachemeson, \& Hansson, 1977), stress fractures (Milner, Ferber, Pollard, Hamill, \& Davis, 2006) and subchondral bone stiffening (Radin, Il, \& Rose, 1973). Although many risk factors associated with overuse injuries have been identified (e.g., age, sex), the most commonly cited, in athletes, are high volume, duration and intensity of activity (Korpelainen, Orava, Karpakka, Siira, \& Hulkko, 2001; Kadel, Teitz, \& Kronmol, 1992).

While the relationship between training volume, repetition and injury has been established, the accurate quantification of these exposures remains elusive. Impact exposure has traditionally been quantified through self-reported mileage (Korpelainen et al. 2001) or by the approximate length of training sessions (Kadel et al. 1992). However, these methods may lead to inaccurate measures of impact exposure given the variation of individual stride lengths due to height, sex and weight (Samson et al, 2001) and the limitations associated with self-reporting methods (Sallis \& Saelens 2000). While accelerometers have also been proposed as a method for quantifying frequency, duration and intensity of lower extremity impacts, they can be costly to implement and require some level of signal processing.

Although pedometers do not distinguish between impact types, they are an attractive alternative for quantifying the impact volume an individual is exposed to given their ease of use, accuracy and affordability (Smith \& Schroeder 2008; Tudor-Locke et al. 2004). While pedometers have traditionally been used as a means to quantify the amount of daily ambulatory physical activity performed by a variety of populations (Tudor-Locke et al. 2009; Zhang, Ohta, Ishikawa- 
Takata, Tabata, \& Miyashita, 2003), they have not been tested as a method of estimating the total number (volume) of impacts experienced by an athlete over a season. However, collecting pedometer data directly from an athlete is a challenging task, as most sporting bodies do not allow external instrumentation during scheduled games.

Therefore, the purpose of the present study was to test the feasibility of utilizing pedometer data collected during gamelike practices to quantify the number of impacts experienced by varsity athletes. If this method is feasible for practices, it may be possible to quantify the total number of impacts experienced by athletes over a season, by knowing the amount of time played by each athlete. This information could advance the study of injury risk associated with impact exposure on a large scale in epidemiological studies.

\section{Methods}

\subsection{Participants}

Forty-four varsity athletes from the women's and men's basketball and soccer teams (mean (SD) age: 20.2 (2.4) years; height: $1.8(0.13) \mathrm{m}$; body mass: $73.3(11.5) \mathrm{kg}$ ) at the University of Windsor provided written informed consent to participate in this study. All procedures were approved by the university's Research Ethics Board.

\subsection{Procedures}

Researchers attended two regularly scheduled practices at two different times (approximately one month apart from each other) throughout the basketball and soccer seasons. Practices were chosen that were anticipated to have running and jumping exposures similar to games and were selected in consultation with each of the head coaches to ensure that two practices of similar intensity and workload were attended. It was ensured that these practices would consist of minimal drills and instead would be focused on activities consistent with game activity, such as running through plays (i.e., scrimmages), time on the sidelines and time receiving instructions from the coach. At each of the practices, participants were equipped with a pedometer (SW-700 Yamax Digi-Walker Fit Solutions, Inc., Optimal Health Products and Services, San Antonio, Texas) that was secured to the waistband over the right hip. These specific pedometers were chosen for this investigation as they have been found to report a valid step count (Leicht \& Crowther, 2009; Arnett \& Lutz, 2002) and they are relatively inexpensive (an important consideration when proposing to outfit an entire sports team). An assumption made here is that all steps recorded by the pedometer represent an impact to the lower extremity, regardless of magnitude, and therefore provide a measure of impact volume. Once securely attached, each pedometer was zeroed and the time at which the pedometer was put on was recorded for each participant (start time); participants wore the pedometer for the duration of the practice. At the conclusion of the practice, the pedometers were removed by the researchers, at which point, the total number of steps and end time were recorded. The start and end times were used to calculate the total time the pedometer was worn by the participant. In the rare event that a pedometer was dislodged from a participant's waistband, the time of occurrence (representing the end time) and the number of steps were recorded. Dislodged pedometers were not reattached to the participants' waistbands to avoid causing interruption to the scheduled practice.

The step data collected from the first practice were normalized to the number of minutes the pedometer was worn, resulting in a steps/minute value for each participant. This normalized step count was then used to estimate the number of steps taken in the second practice (estimated step count (ES)) by multiplying the normalized step count from practice one by the length of the second practice. The ES was compared to the pedometer reading from the second practice (actual step count (AS)) as a measure of accuracy.

Finally, to demonstrate how this method could be utilized to estimate the number of impacts experienced over an entire season, publicly available data regarding the total playing time for each basketball athlete over a competitive season (Ontario University Athletics, 2010), were multiplied by their normalized step count.

\subsection{Statistical Analysis}

As a measure of pedometer reliability and to ensure that two similar practices were attended, a three-way ( 2 sex $\mathrm{x} 2$ sport $\mathrm{x} 2$ practice) mixed repeated-measures ANOVA was used in combination with a Pearson correlation analysis. It should be noted that the data from dislodged pedometers were removed from this initial analysis, but were included subsequently. A three-way ( 2 sport x 2 sex x 2 method (actual steps vs. estimated steps)) mixed repeated measures ANOVA with sex and sport as the between-subject variables and Pearson correlation analysis were used to compare the ES and AS step counts from practice two. To further assess the accuracy of the method presented here, a Bland-Altman test was also performed (Bland and Altman, 1986). The absolute and relative (\%) differences between ES and AS were calculated. To assess the effect of sport and sex (independent variable) on the absolute ES-AS differences (dependent variable), a two-way ANOVA ( 2 sport x 2 sex) was used. All statistical analyses were performed using SPSS-PASW 18.0 (SPSS Inc., IBM, Chicago, IL) and significance was assessed at $\alpha<0.05$.

\section{Results}

Overall, the pedometers were worn by each participant for a mean (SD) time of 105 (23) minutes and 112 (22.3) minutes for practice one and two, respectively, and only $7.8 \%$ of the pedometers experienced a dislodgement. A significant moderate relationship $(\mathrm{r}=0.52)$ was calculated for the number of steps between practices and there were no significant mean (SD) step count differences reported between practice one and two $(F=3.95 ; p=0.07)$ (Figure 1). There was a significant main effect of sport, where the soccer athletes took significantly more steps than their basketball counterparts $(\mathrm{F}=7.81 ; \mathrm{p}=0.008)$ (Figure 1). Overall, there was no significant difference between the mean ES and AS examined $(\mathrm{t}=0.970 ; \mathrm{p}=0.34)$ and there were no sport by method or sex by method interaction effects discovered. 


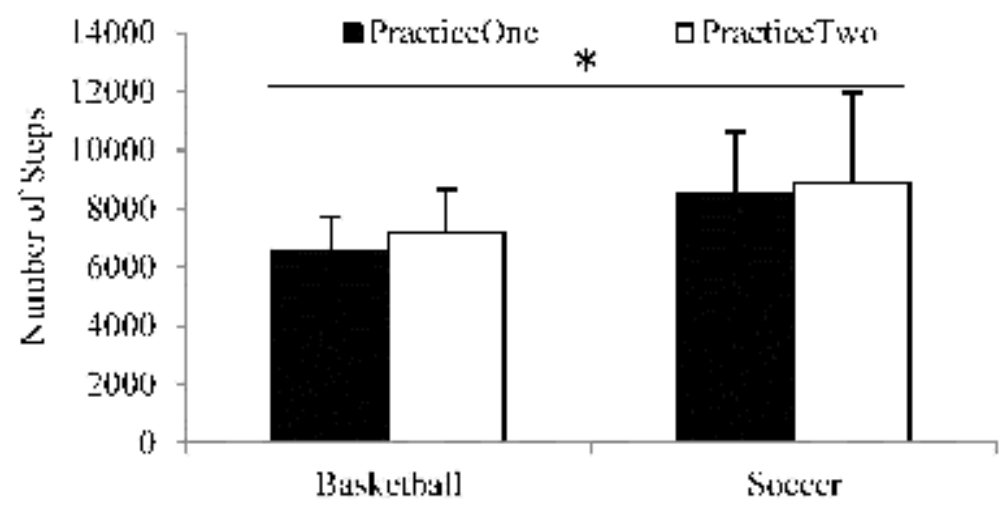

Figure 1. Comparison of the mean (SD) step counts between practice one and practice two across sports $(* \mathrm{p}<0.05)$

A Pearson correlation coefficient of $0.72(\mathrm{p}<0.05)$ suggests a strong relationship between ES and AS (Figure 2a) across sports. With mean absolute differences ranging from 7 to 3958 steps the Bland-Altman plot suggests that this was an accurate method with more than $95 \%$ of the values falling within plus/minus two standard deviations of the mean difference (Figure 2b).

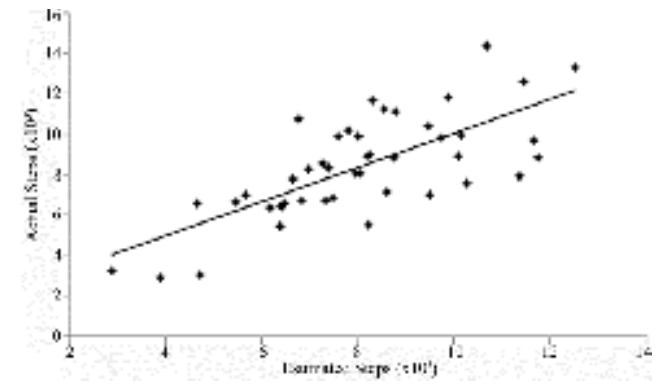

(a)

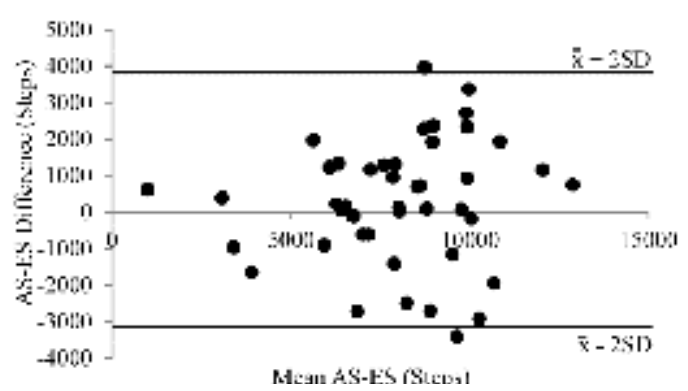

(b)

Figure 2. (a) Scatterplot showing the relationship between actual steps (AS) and estimated steps (ES) for all participants $(\mathrm{r}=0.7)$ and $(\mathrm{b})$ the results of a Bland-Altman analysis.

There was a significant ( $\mathrm{p}=0.008)$ sport main effect on the mean (SD) absolute difference, such that soccer participants had a mean step difference that was 1.7 times greater than basketball participants (1848 (1165) vs. 1056 (875)) (Figure 3 ). In comparison, the absolute difference between ES and AS with respect to sex was not significant ( $\mathrm{p}=0.80)($ Figure 3).

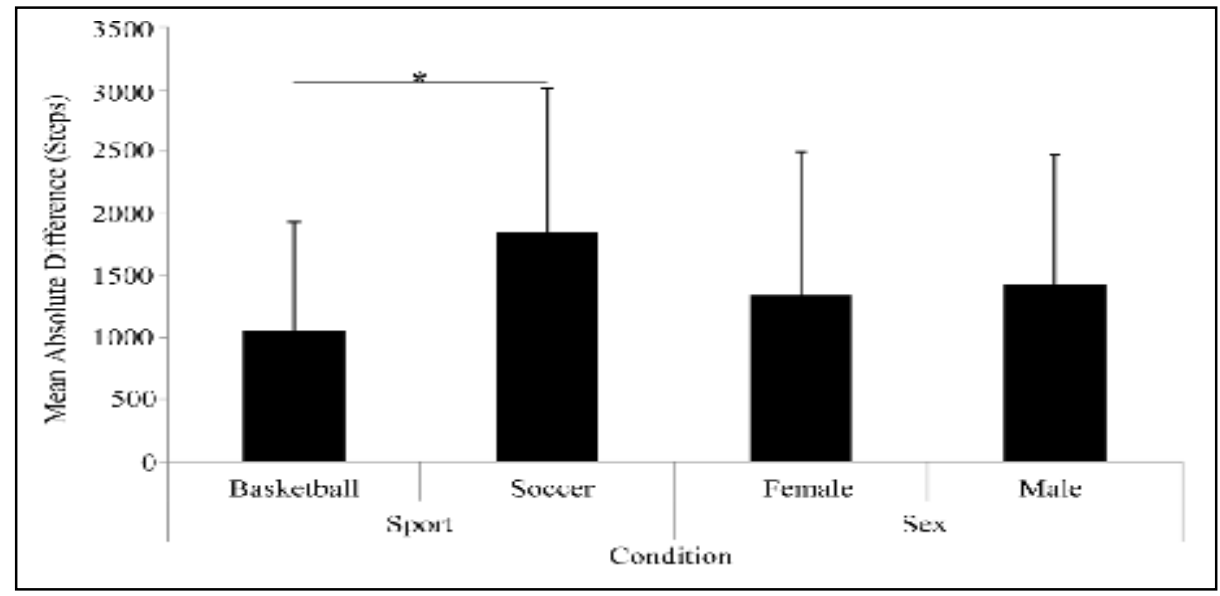

Figure 3. Mean (SD) absolute differences between the estimated and actual steps of male and female athletes from the basketball and soccer teams $(* \mathrm{p}<0.05)$. 
The mean relative difference between ES and AS did not exceed $21 \%$ across sex or sport (Figure 4). Mean relative differences ranged from $11.0 \%$ to $20.7 \%$, with women's soccer and basketball participants yielding the highest and lowest differences between ES and AS, respectively (Figure 4).

Figure 4. Mean

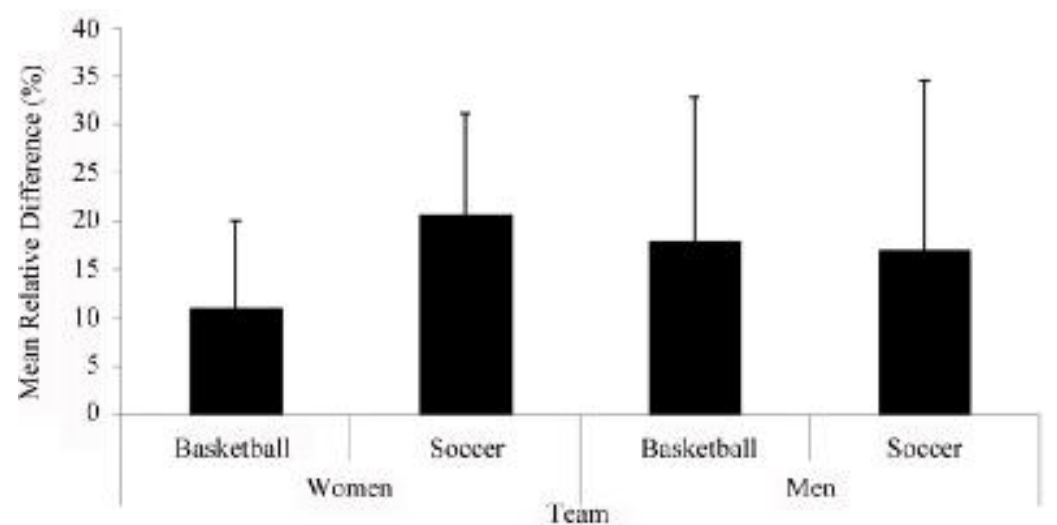

differences between the estimated and actual steps of male and female athletes from the basketball and soccer teams.

Across all participants, the range of relative differences was $0.1 \%$ to $53.8 \%$, with approximately $75 \%$ of the participants exhibiting errors which were below 25\% in magnitude (Figure 5). No significant main or interaction effects were found.

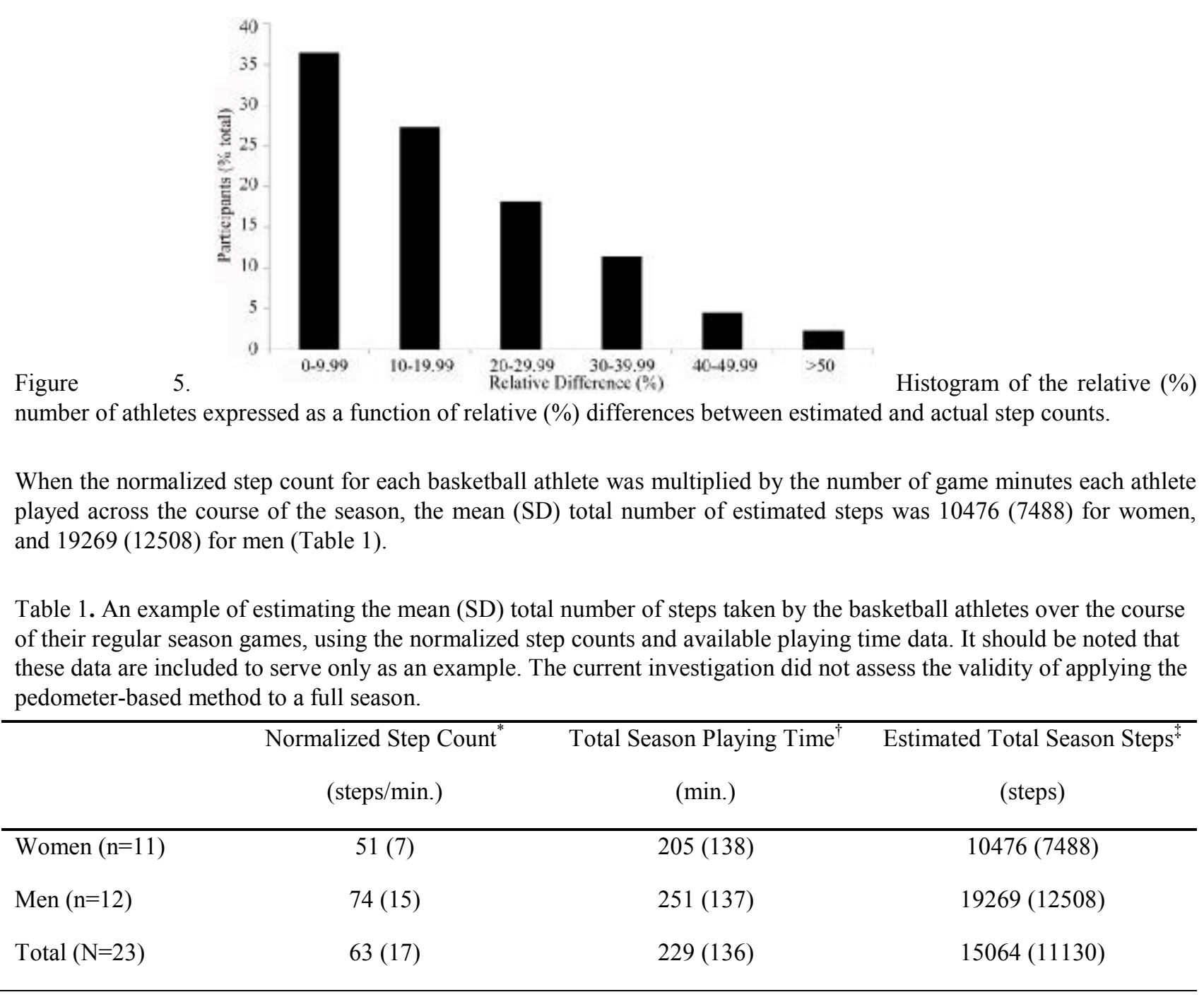

*From pedometer data and playing time during first practice

${ }^{\dagger}$ From Ontario University Athletics Association website (www.oua.ca)

${ }^{\ddagger}$ Determined as the normalized step count multiplied by season playing time 


\section{Discussion}

To the authors' knowledge, this is the first study to examine the use of pedometers as a method of estimating the total number of steps of varsity athletes during practices. The presented results suggest that calculating a normalized pedometer-based step count from a practice is a feasible method for calculating total impact volume for subsequent events with running and jumping exposure. The reasonably low mean relative difference between the ES and AS, the absence of significant differences, the strong relationship between the ES and AS across all participants and the relative simplicity of this approach, highlight how useful this method could be for estimating the total impact volume experienced by university athletes.

The data presented here show that basketball and soccer athletes competing at the university level are taking a large volume of steps during practices. For example, basketball and soccer athletes had mean step counts that would categorize them as somewhat active (7500 - 9999 per day) to active ( $>10000$ per day) based on only two hours of practice time (Tudor-Locke \& Bassett, 2004). In terms of exercise intensity, the normalized step counts would suggest that both sets of athletes were performing at light exercise intensity ( $<96$ steps per minute) (Tudor-Locke et al., 2005). This is an overall approximation based on characteristics of these sports, in which the participants experience short bouts of high intensity exercise (e.g., running, jumping), followed by more prolonged periods of low intensity (e.g., walking) activities.

In the current investigation, the playing time (i.e., the time each player is involved in competition), as opposed to real time (i.e., the total time of the competitive event including all play stoppages), was used to calculate the total seasonal step count for basketball players, as an example of how this approach could be used. While steps could still be occurring during non-playing times, the relative contribution of these steps may be assumed to be relatively low, and therefore, the total seasonal step count provides an estimate of the total number of steps that would occur. Using game times also eliminates the probability of falsely calculating a step when the player may be stationary (e.g., sitting on the bench, resting during half-time).

Soccer athletes tended to have a significantly greater ES-AS difference compared to basketball athletes, suggesting that the type of sport has an effect on the impact exposure estimating method presented here. This finding may be a result of the differences in the variations of the steps performed within each of the respective sports (Gil, Gil, Ruiz, Irazusta, \& Irazusta, 2007; Crisafulli et al., 2002). In general, the greater number of unique positions in soccer results in a greater variability between athletes with respect to the distance each player travels (Gil et al. 2007), compared to basketball players, who, while playing different positions, generally travel comparable distances (Crisafulli et al. 2002). This disparity may introduce inconsistencies in step counts between practices within each of the sports. Future studies need to consider a method of accounting for the type of sport that is being analysed when evaluating the viability of this approach over longer periods.

With respect to the type of impacts experienced by different athletes, basketball players are generally exposed to both running and jumping initiated impact activities, whereas soccer players primarily experience running initiated impacts only. While this may help to explain the differences between sports, it also highlights a limitation of using pedometers to quantify impact exposure; a pedometer records only that a step has occurred and not the intensity of the step. Previous research suggests that forces in the lower extremity during jumping can exceed 10 times body weight (McNair \& Prapavessis 1999), as opposed to running, in which forces may reach only 3 times body weight (Nigg et al. 1995). While the magnitude of the impacts is an important factor in predicting injury, the aim of this work was to quantify only the number of impacts, with a simple, cost-effective and readily available technology.

While the current investigation demonstrates the feasibility of a pedometer-based method for tracking the number of impacts experienced by an university athlete, this method is based on the assumption the activities performed during a practice accurately reflect those that would take place during a competition. Although precautions were taken (i.e., consultation with head coaches) to collect data from practices that closely matched the movements and intensity of games, it is likely that practice-game differences would affect the accuracy of this method. This highlights the importance of consulting with coaches to ensure that normalization data are collected from practices that most closely resemble the structure of a game. Including data from a greater number of practices may help to improve on this limitation (Clems \& Griffiths 2008). Furthermore, the current method does not account for impact frequency, which may also be a contributing factor of distal lower extremity injuries and may differ between games and practices.

Along with a feasibility analysis, an example of using this method to quantify the number of impacts experienced over an entire season was provided and was included to highlight the ease of use of this method. The majority of sporting bodies track and provide each athlete's playing time for each game as a part of their statistics reporting. Theoretically, this is the only information, aside from the normalized step counts from practices that are required, to quantify total seasonal step counts. It deserves further mention that the accuracy of the method presented here has only been assessed with respect to practices and has not been validated to calculate step volume over an entire season. Therefore, establishing the validity of this method for estimating total impact exposure over an entire season should remain a priority of future studies. To accomplish this, a protocol will need to be developed that allows the direct counting of steps taken during a game (e.g., physically counting the number of steps from video analysis), as most sport governing bodies do not allow external instrumentation to be worn during competition. Finally, the current method should be extended to include data from more practices, and to enhance the generalizability of the approach, different sports should also be assessed. 
Overall, the errors between the actual number of steps measured by a pedometer and those that were predicted using the pedometer-based time normalization method were relatively small. These errors, combined with the ease of use and low cost of pedometers, suggests that pedometers are a feasible tool to predict the total volume of lower extremity impacts experienced by varsity basketball and soccer athletes on a large scale.

\section{Acknowledgements}

We would like to thank NSERC for funding this project. Additional thanks to coaches Chantal Vallée, Chris Oliver, and Steve Hart, and to the University of Windsor women's and men's varsity basketball and soccer teams.

\section{References}

Arnett, M. G., \& Lutz, B. (2002). Effects of rope-jump training on the os calcis stiffness index of postpubescent girls. Medicine and Science in Sport and Exercise, 34, 1913-1919.

Bland, J. M., \& Altman, D. G. (1986). Statistical methods for assessing agreement between two methods of clinical measurement. Lancet, 327, 307-310.

Clems, S. A., \& Griffiths, P. L. (2008). How many days of pedometer monitoring predict monthly ambulatory activity in adults? Medicine and Science in Sport and Exercise, 40, 1589-1595.

Crisafulli, A., Melis, F., Tocco, F., Laconi, P., Lai, C., Concu, C. (2002). External mechanical work versus oxidative energy consumption ratio during basketball field test. Journal of Sports Medicine and Physical Fitness, 42, 409-417.

Gil, S. M., Gil, J., Ruiz, F., Irazusta, A., Irazusta, J. (2007). Physiological and anthropometric characteristics of young soccer players according to their playing position: Relevance for the selection process. Journal of Strength and Conditioning Research, 21, 438-445.

Kadel, N. J., Teitz, C. C., Kronmol, R. A. (1992). Stress fractures in ballet dancers. American Journal of Sport Medicine, 20, 445-449.

Korpelainen, R., Orava, S., Karpakka, J., Siira, P., Hulkko, A. (2001). Risk factors for recurrent stress fractures in athletes. American Journal of Sport Medicine, 29, 304-310.

Leicht, A. S., \& Crowther, R. G. (2009). Influence of non-level walking on pedometer accuracy. Journal of Science and Medicine in Sport, 12, 361-365.

McNair, P. J., \& Prapavessis, H. (1999). Normative data of vertical ground reaction forces during landing from a jump. Journal of Science and Medicine in Sport, 2, 86-88.

Milner, C. E., Ferber, R., Pollard, C. D., Hamill, J., Davis, I. S. (2006). Biomechanical factors associated with tibial stress fractures in female runners. Medicine and Science in Sport and Exercise, 38, 323-328.

Nigg, B. M., Cole, G. K., Bruggemann, G. (1995). Impact forces during heel-toe running. Journal of Applied Biomechanics, 11, 407-432.

Ontario University Athletics. (2010). Retrieved from www.oua.ca.

Radin, E. L., Il, P., Rose, R. M. (1973). Studies of joint function. Journal of Bone and Joint Surgery, 55, $1303-1303$.

Sallis, J. F., \& Saelens, B. E.(2000). Assessment of physical activity by self-report: status, limitations, and future directions. Research Quarterly for Exercise and Sport, 71, 1-14.

Samson, M.M., Crowe, A., de Vreede, P. L., Dessens, J. A. G., Duursma, S. A., Verhaar, H. J. J. (2001). Differences in gait parameters at a preferred walking speed in healthy subjects due to age, height and body weight. Aging Clinical and Experimental Research, 13, 16-21.

Serink, M. T., Nachemson, A., Hansson, G. (1977). The effect of impact loading on rabbit knee joints. Acta Orthopaedica Scandinavica, 48, 250-262.

Smith, J. D., \& Schroeder, C. A. (2008). Assessing pedometer accuracy while walking, skipping, galloping, sliding and hopping. Journal of Strength and Conditioning Research, , 22, 276-282.

Tudor-Locke, C., \& Bassett, D. R. (2004). How many steps/day are enough? Preliminary pedometer indices for public health. Sports Medicine, 34, 1-8.

Tudor-Locke, C., Bell, R. C., Myers, A. M., Harris, S. B., Ecclestone, N. A., Lauzon, N., Rodger, N. W. (2004). Controlled outcome evaluation of the first step program: A daily physical activity intervention for individuals with type 2 diabetes. International Journal of Obesity, 28, 113-119.

Tudor-Locke, C., Sission, S. B., Collova, T., Lee, S. M., Swan, P. D. (2005). Pedometer-determined step count guidelines for classifying walking intensity in a young ostensibly healthy population. Canadian Journal of Applied Physiology, 30, 666-676.

Tudor-Locke, C., Sisson, S. B., Lee, S. M., Craig, C. L., Plotnikoff, R. C., Bauman, A. (2009). Evaluation of quality of commercial pedometers. Canadian Journal of Public Health, 7, 10-15.

Zhang, J. G., Ohta, T., Ishikawa-Takata, K., Tabata, I., Miyashita, M. (2003). Effects of daily physical activity recorded by pedometer on peak oxygen consumption (VO2 peak), ventilatory threshold and leg extension power in 30 to 69 year old Japanese without exercise habit. European Journal of Applied Physiology, 90, 109-113. 